\title{
Changes in Cancer-Related Risk Perception and Smoking Across Time in Newly-Diagnosed Cancer Patients
}

\author{
Jennifer L. Hay, ${ }^{1,3}$ Jamie Ostroff, ${ }^{1}$ Jack Burkhalter, ${ }^{1}$ Yuelin Li, ${ }^{1}$ \\ Zandra Quiles, ${ }^{1}$ and Alyson Moadel $^{2}$
}

Accepted for publication: January 26, 2007

Published online: March 2, 2007

We examine the bidirectional relationships between cancer risk perceptions and smoking be-
havior among newly diagnosed cancer patients $(N=188)$ during hospitalization for surgical
resection, and at three and 12 months subsequently. Those with higher perceptions of risk
for developing another cancer at three months were most likely to abstain from smoking
by twelve months. Patients were relatively accurate in their cancer risk perceptions, with re-
lapsers and continuous smokers reporting higher levels of risk perceptions at twelve months.
Finally, those who quit smoking by 12 months felt at lower risk for developing cancer by
12 months. None of these relationships were significant between baseline and three months.
Results indicate that perceived risk of cancer recurrence may be clinically useful in motivat-
ing smoking cessation after the acute cancer treatment phase is over. This study justifies an
expanded theoretical framework attending to the distinct, prospective influences of illness
risk perceptions on health behavior, and of health behavior on illness risk perceptions.

KEY WORDS: risk perceptions; health behavior theory; smoking cessation.

The perception of illness risk, defined as an individual's belief about his or her chances of becoming ill, is one of the most frequently cited constructs in health behavior change theories (e.g., Ajzen and Fishbein, 1980; Janz and Becker, 1984; Leventhal and Cameron, 1987; Rogers, 1983; Sutton, 1982; Weinstein, 1988). These theories universally propose that increased illness risk perceptions are related to the adoption of health protective actions, and thus require the use of prospective research designs in order to examine the effect of a prior attitude (risk perception) on subsequent behavior change (Gerrard et al., 1996a, 1996b; Weinstein et al., 1998; Weinstein and Nicolich, 1993). This relationship has been widely

\footnotetext{
${ }^{1}$ Memorial Sloan-Kettering Cancer Center, Department of Psychiatry and Behavioral Sciences, 641 Lexington Avenue, 7th Floor, New York, NY 10022, USA.

${ }^{2}$ Albert Einstein College of Medicine, Department of Epidemiology and Population Health, 1300 Morris Park Avenue, Bronx, NY 10461, USA.

${ }^{3}$ To whom correspondence should be addressed; e-mail: hayj@ mskcc.org.
}

examined in regard to a wide range of health protective actions, including the adoption of safer sex strategies to prevent transmission of human immunodeficiency virus (see Gerrard et al. for a review), cancer screening behaviors (see Vernon, 1999 for a review), and smoking cessation (Norman et al., 1999). However, given that the diffusion of a health protective action is an ongoing process, illness risk perceptions are also likely to change subsequent to the adoption of health protective actions. There is a limited literature documenting this (Aiken et al., 1995; Brewer et al., 2004; Gerrard et al., 1996a, 1996b; Gibbons et al., 1991).

These findings provide an opportunity to expand existent theoretical frameworks to include a more complete set of expectations concerning the relationships between illness risk perceptions and the adoption of health protective behaviors. In his Social Cognitive Theory, Albert Bandura (1986) endorsed "reciprocal determinism," such that health cognitions and health behaviors each affect each other over the course of time, and that only by 
understanding this dynamic can we understand behavior change maintenance. Bandura proposed that self-efficacy for performing a behavior promotes occurrence of that behavior, and that the performance of the behavior, in turn, subsequently enhances self-efficacy. In the context of risk perceptions, Brewer and colleagues (2004) have clarified three distinct, mutually exclusive hypotheses encompassing the relationships between illness risk perceptions and behavior. These hypotheses form the basis for theoretical expansion of the process by which illness risk perceptions and behavior affect each other bidirectionally over time. These hypotheses are described below.

\section{The Behavior Motivation Hypothesis}

As described previously, this hypothesis asserts that risk perceptions lead individuals to adopt health protective actions. This is the hypothesis that is welldescribed in health behavior change theories. This hypothesis can be evaluated only prospectively, with a measure of risk perception at one time point and behavior at a later time point. This hypothesis is important because it identifies risk perceptions as critical precursors of subsequent behavior change.

\section{The Accuracy Hypothesis}

This hypothesis asserts that people who are engaging in risk behaviors have higher risk perceptions at any given time point. This is the only hypothesis that can be assessed with a cross-sectional bivariate statistic (Brewer et al., 2004). Evidence for this hypothesis regarding smoking behavior is wellestablished, in that smokers' illness risk perceptions exceed that for non-smokers (Weinstein, 1999). An example of the type of thinking implied by the accuracy hypothesis is, "I know that as a smoker, my risk of developing cancer is higher than the risk for people who do not smoke." This hypothesis addresses the extent to which people are correctly or incorrectly assessing their current risk status.

\section{The Risk Reappraisal Hypothesis}

Finally, this hypothesis asserts that if an action is taken to decrease the risk of becoming ill, then risk perceptions should decrease subsequent to behavior change. Again, this hypothesis can only be assessed prospectively, with risk perception AND behavior assessed at an initial date and then reassessed at a later date. Two studies have examined this hypothesis in the cancer context. Gibbons and colleagues (1991) found that smokers who quit after smoking cessation treatment reduced their smokingrelated health risk perceptions. This study found that relapsers reduced their risk perceptions even more than did abstainers, indicating some defensive denial of their risks. Aiken and colleagues (1995) found evidence that the adoption of mammography led to reductions in perceived risk for breast cancer. While screening does not actually reduce future risk, this study provides additional substantiation of a common process - adoption of health protective actions can lead to changes in illness risk perceptions. Further examination of this hypothesis is important because it may provide explanations for maintenance over time, given that reduced risk perceptions may serve as an ongoing impetus to continue health protective behaviors, such as smoking cessation or repeat cancer screening.

Taken together, careful assessment of all three of these predicted relationships dictated by the behavior motivation, accuracy, and risk reappraisal hypotheses provide the opportunity to examine the dynamic interplay of perceived risk and behavior change over time.

\section{The Current Study}

The goal of this study is to examine these three proposed relationships between cancer-related risk perceptions and smoking behavior (behavior motivation, accuracy, and risk reappraisal) over the course of one year among patients recently diagnosed with lung and head and neck cancers. We believe this sample provides a unique opportunity to examine these factors across time, due to the salience of thoughts about health risks and recurrence among patients diagnosed with tobacco-related cancers, and the fact that these perceptions may relate to diverse patterns of tobacco consumption after cancer diagnosis (McBride and Ostroff, 2003). Schnoll and colleagues (2003) examined whether cancer-related risk perceptions influenced subsequent smoking behavior in those with tobacco-related cancers. These authors found evidence for the behavior motivation hypothesis, such that lung and head and neck cancer patients with higher cancer-related risk perceptions were more likely to have quit smoking 
three months later. In an earlier cross-sectional paper examining the same sample, Schnoll and colleagues (2002) found that lung and head and neck cancer patients who quit smoking had higher cancer-related risk perceptions than those who continued to smoke, providing no evidence for the accuracy hypothesis. The current study will extend this work by examining three reciprocal, mutually exclusive relationships between cancer-related risk perceptions and smoking behavior patterns, as proposed by Brewer and colleagues (2004), over 12 months among patients diagnosed with tobacco-related cancers.

Clinically, this study may help us understand whether cancer risk perceptions are of the greatest motivational significance at the time of cancer diagnosis and treatment, or later as treatment demands abate, as well as how risk perceptions may differentially impact diverse patterns of smoking and quitting after cancer diagnosis. Theoretically, the study may provide further evidence for the value in addressing the multiple relationships between risk perceptions and behavior change across time within existent health behavior theories.

\section{METHOD}

\section{Participants}

Patients newly diagnosed with either head and neck or lung cancer were recruited to this study. Eligibility criteria included all patients treated at Memorial Sloan-Kettering Cancer Center who: (1) were diagnosed with either (a) squamous cell carcinoma of the oral cavity, pharynx and larynx, or (b) Stage I, II, IIIa non-small cell lung carcinoma; (2) had no evidence of distant metastatic disease; (3) were diagnosed within the prior six months; (4) were at least 18 years of age; (5) had evidence (from patient self-report or medical chart notes) of tobacco use within 6 months prior to cancer diagnosis; (6) were English speaking; and (7) provided informed consent. The study was approved by the medical center's human subjects review board. Eligible patients were identified by weekly medical screening for eligibility through the hospital's computerized disease management system, and by referrals from medical staff. Most eligible patients were identified and recruited for participation while they were inpatients undergoing surgical treatment $(62.2 \%)$. The remaining patients $(37.8 \%)$ were recruited as outpatients (e.g., post-surgery or receiving chemotherapy and/or radiation therapy). Of the 215 eligible patients approached for accrual, 188 (87.4\%) lung and head and neck cancer patients provided informed consent and constitute the final sample.

Table I shows that the sample consisted of primarily lung cancer patients $(65 \%)$, and the remainder had head and neck cancer $(35 \%)$. Most patients' diseases were at Stages I (40\%) and II (38\%). The majority of the patients were Caucasian (92\%). Greater than half of the sample had completed at least some college and had an income of $\$ 50,000$ or above.

Of the patients who did not participate $(N=27)$, reasons provided for refusal included being too ill, not wanting to be bothered, and being anxious or avoidant. Most of the sample (62\%) completed the baseline assessment during hospitalization. The remaining patients completed their baseline assessments after hospitalization.

\section{Procedure}

A trained research assistant approached eligible patients to offer them participation. The consenting process was always in person, and no monetary compensation was offered. Efforts were made to establish rapport and create a comfortable environment that respected confidentiality and included

Table I. Characteristics of the Patient Sample

\begin{tabular}{lc}
\hline \multicolumn{1}{c}{ Characteristics } & Statistics $N=188$ \\
\hline Gender (female) & $96(51 \%)$ \\
Age (yrs. mean, sd) & 61 yrs $(10)$ \\
Ethnicity (white) & $172(92 \%)$ \\
Education (partial college or above) & $110(59 \%)^{a}$ \\
Income (above 50,000/year) & $87(52 \%)^{b}$ \\
Cancer site & \\
$\quad$ Lung cancer & $122(65 \%)$ \\
Head and neck cancer & $66(35 \%)$ \\
Primary tumor staging & \\
Stage I & $71(40 \%)$ \\
Stage II & $68(38 \%)$ \\
Stage III & $21(12 \%)$ \\
Stage IV & $17(10 \%)$ \\
Tobacco use (7-day point abstinence) & \\
Baseline & $162(87 \%)$ \\
3 Months & $142(84 \%)^{d}$ \\
12 Months & $119(79 \%)^{e}$ \\
\hline
\end{tabular}

Note. Statistics are $\mathrm{N}$ and percentages unless otherwise noted.

$a_{2}$ did not respond.

${ }^{b} 8$ did not respond.

${ }^{c} 11$ did not respond (or did not know), $n=177$ was the denominator.

${ }^{d} 19$ missing, $n=169$ was the denominator.

e 38 missing, $n=150$ was the denominator. 
assurances that any tobacco use information that the patient provided would not be reported to the patient's physician or other health care team members. Most assessments were conducted by face-to-face interview and took approximately $45 \mathrm{~min}$ to complete. In a small number of cases, after providing consent, patients completed the questionnaire at home and returned it by mail, or a telephone interview was conducted. Follow-up assessments were administered at a clinic appointment or by telephone 3 and 12 months later. Data from patients were collected on numerous domains, including sociodemographics, smoking history, medical events, physical symptoms, psychological distress, and social support for quitting. The present analyses will focus on cancer-related risk perceptions and smoking behavior across time.

\section{Measures}

\section{Cancer-Related Risk Perception}

Questions that assess a person's future risk of becoming ill and omit any behavioral intentions can confound risk perception with intentions. Thus when asked about her risk of developing another lung cancer, a patient may feel her risk is lower than other lung cancer survivors if she intends to quit smoking immediately. Therefore, questions assessing cancer-related risk perceptions should include a conditional behavior statement if responses are used to predict subsequent behavior change (e.g., Ronis, 1992). Therefore, head and neck cancer patients were asked, "If you were to continue smoking, what do you think your chances are of developing another head and neck cancer? These patients were also asked the same question concerning their chances of developing lung cancer, as well as any other form of cancer (Gibbons et al., 1991). As such, head and neck cancer patients were asked three questions concerning their cancer risk perceptions. Lung cancer patients were not asked their risk of head and neck cancer because of low awareness of these cancers in the general population (Horowitz et al., 1995; Warnakulasuriya et al., 1999); therefore lung cancer patients were asked only two questions concerning their risk perceptions for developing another lung cancer, or any other form of cancer. All responses were measured on a 5-point Likert-type scale ranging from 1 (not likely) to 5 (extremely likely). The mean of the items was used as the conditional risk score (alpha reliability $=0.92$ ) to test the behavior motivation hypothesis.
We used a parallel set of risk perception questions that dropped the conditional statement, "What do you think your chances are of developing the following... (a) another head and neck cancer, (b) lung cancer, and (c) any other form of cancer," $($ alpha reliability $=0.83$ ) to test the accuracy and risk reappraisal hypotheses because these hypotheses reflect attitudinal responses to actual - rather than future - behavior, i.e., what are your chances of developing cancer given your current smoking behavior (Brewer et al., 2004). This parallel set of questions that dropped the conditional statement were asked after the conditional risk questions.

We examined the extent to which context variables (hospital vs. outpatient recruitment, face-toface, mail, and telephone assessment) were related to baseline levels of conditional and general risk perceptions, respectively. No statistically discernible differences were found (all $p \mathrm{~s}>0.05$ ).

\section{Tobacco Use}

We assessed self-reported 7-day point abstinence at three time points (baseline, 3 months, and 12 months). Additionally, we biochemically confirmed 3 and 12-month self-reported abstinence using urine cotinine, and we corrected our point abstinence data for inconsistent reports (the disconfirmation rate was $2.9 \%$ at 3 months and $2.6 \%$ at 12 months). Based on available data, 7-day point-abstinence rates were $87 \%$ at baseline, $84 \%$ at 3 months, and $79 \%$ at 12 months. As such, we identified patients as continuous smokers if they were smoking at baseline and again at 3 months. Late quitters included patients that smoked at baseline but were abstinent at 3 months. Patients were considered relapsers if they were abstinent at baseline but smoking at 3 months. Finally, we identified abstainers as those who were abstinent at baseline and 3 months. We identified these individuals as abstainers rather than continuous abstainers because we could not rule out slips to smoking between assessment periods (Hughes et al., 2003). The 12 -month profiles were created in parallel fashion based on 7-day point abstinence at 3 and 12 months. We used participants' reports of 7-day point abstinence to calculate 3-month smoking behavior profiles first, because this is an established algorithm to examine prospective smoking behavior in cancer patients (Ostroff et al., 2000; Schnoll et al., 2003), and second, because we thought that smoking behavior change patterns over an extended time 
interval (i.e., a number of months) had more important implications for intervention than smoking behavior over the most recent 7 days prior to assessment (e.g., Hughes et al., 2003).

\section{Physical Status}

We also included assessments of patients' stage of disease assessed at baseline, as well as physical symptoms and medical events over the 1-year period as potential covariates of the risk-smoking behavior relationship. These factors could influence patients' assessments of their cancer-related risk perceptions after cancer diagnosis. The Physical Symptoms subscale from the Memorial Symptom Assessment Scale (MSAS; Portenoy et al., 1994) was used to assess the existence of physical symptoms associated with cancer and its treatment. The subscale assesses the extent to which the following symptoms (lack of appetite, lack of energy, pain, feeling drowsy, constipation, dry mouth, nausea, vomiting, change in taste, weight loss, feeling bloated, dizziness) bothered or distressed them in the past week on a 5-point scale, ranging from 0, "not at all" to 4, "very much." Internal consistency for the Physical Symptoms subscale is high in our sample (Cronbach $\alpha$ coefficient $=$ .77). We assessed physical symptoms at baseline, 3 and 12 months. The mean physical symptom summary scores (range 0 to 48$)$ were $14.7(S D=8.2)$ at baseline, $10.5(S D=7.8)$ at 3 months, and 8.1 $(S D=7.2)$ at 12 months.

We computed two medical event scores - one for interim cancer diagnoses and one for other medical events experienced since the last assessment. $\mathrm{Pa}$ tients reported cancer recurrences or new primary cancer diagnoses (yes vs. no) between baseline and 3 months, and then between 3 and 12 months. At 3 and 12 months respectively, $2.5 \%$ and $12 \%$ of patients reported cancer recurrences or new primary diagnoses. We also assessed the occurrence of 7 other medical events (planned hospitalizations, unplanned hospitalizations, visits to urgent care, psychiatry consultation, chemotherapy, radiotherapy, diagnosis of another medical illness) that transpired between baseline and 3 months, and 3 and 12 months. At 3 and 12 months respectively, $56.5 \%$ and $36.2 \%$ of patients reported at least one medical event.

Additionally, we included two measures of physiological dependence to tobacco as additional covariates. The Fagerstrom Test for Nicotine Dependence (Fagerstrom et al., 1990; Heatherton et al., 1991) measures dependence to nicotine and has good internal reliability (Cronbach's alpha $=0.61$ ), and predicts biochemical exposure (carbon monoxide and salivary cotinine), withdrawal symptoms, and smoking relapse (Heatherton et al., 1991; Lichtenstein and Mermelstein, 1986). We also measured number of cigarettes smoked per day at baseline. In this sample, average Fagerstrom Test Scores were $5.7(s d=$ 2.2), and average number of cigarettes smoked per day was $31(s d=14)$.

\section{Specific Hypotheses}

We separately examine three hypotheses as suggested by Brewer and colleagues (2004). First, we examine the behavior motivation hypothesis, and predict that those with higher cancer-related risk perceptions at baseline will report abstinence in the subsequent three months, and that those with higher cancer-related risk perceptions at three months will report abstinence in the subsequent nine months. Next, we examine the accuracy hypothesis, and predict that patients who have continued to smoke will have higher cancer-related risk perception at three and 12 months. Finally, we examine the risk reappraisal hypothesis, and predict that those who quit by three months will be more likely to reduce their cancer-related risk perception at three months, and then whether those who quit between three and 12 months are more likely to reduce their cancerrelated risk perception at 12 months. Unless otherwise noted, listwise deletion was used when we did not have complete data on all factors. ${ }^{4}$

\section{RESULTS}

\section{Behavior Motivation Hypothesis}

We first examined whether cancer-related risk perception influenced subsequent smoking behavior. In these analyses, we used a logistic regression model for multinomial response variables (Agresti, 2002; McCullagh and Nelder, 1999). Smoking behavior was the dependent variable and the patients' risk perception was the independent variable. Two separate multinomial models were fitted. The first examined

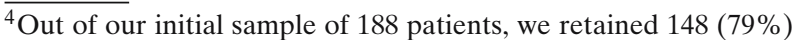
at 12 months. Those who were retained at 12 month assessment did not differ from those who were missing on baseline perceived risk or smoking status (all $p \mathrm{~s}>.05$ ).
} 
whether or not risk perceptions assessed at baseline predicted smoking behavior at 3 months followup, using continued smokers as the reference group. A statistically significant coefficient for risk perceptions indicates that risk perceptions assessed at baseline reliably predicts subsequent smoking behavior at 3 months follow-up. The second multinomial logistic regression tested whether or not risk perceptions assessed at 3 months follow-up predicted smoking behavior at 12 months follow-up. Figure 1 is a visual aide plotted to help interpret the results of the multinomial logistic regression models.

Figure 1 shows how risk perceptions assessed at baseline was associated with smoking behavior at 3 month follow-up (left panel) and how risk perceptions assessed at 3 months follow-up was associated with smoking behavior at 12 months follow-up (right panel). The points on Fig. 1 are the observed proportions of patients in different smoking profiles. The lines are predicted probability of smoking behavior estimated by multinomial models, using smoking status as the dependent variable and risk perceptions as the sole predictor. As such, the left panel of Fig. 1 illustrates that at 3 months follow-up, over $80 \%$ of the patients were abstinent. Patients who had high baseline risk perceptions (e.g., those who gave an average risk near ' 5 ') were as likely to be abstinent from baseline through three-month followup as patients who had lower baseline risk perceptions. The model-predicted lines for other smoking behavior profiles also support this observation, suggesting no strong association between risk perceptions and abstinence at 3 months follow-up. The coefficient associated with risk perceptions was not statistically significant $\left(\chi^{2}[3, N=166]=0.07, p=\right.$ 0.99 ), suggesting that there was no statistically discernible difference between the slopes of the four lines. Risk perceptions at baseline did not predict smoking status at 12 months either.

However, a strong association emerges between risk perceptions and smoking behavior at the later time period. This is seen in the right panel of Fig. 1. The solid line (labeled "Abstainers") shows that the higher the risk perceptions at three months, the more likely the patients are to be abstainers, and less likely to be continuous smokers (the broken line labeled "Continuous Smokers"). This multinomial model shows that risk perceptions assessed at
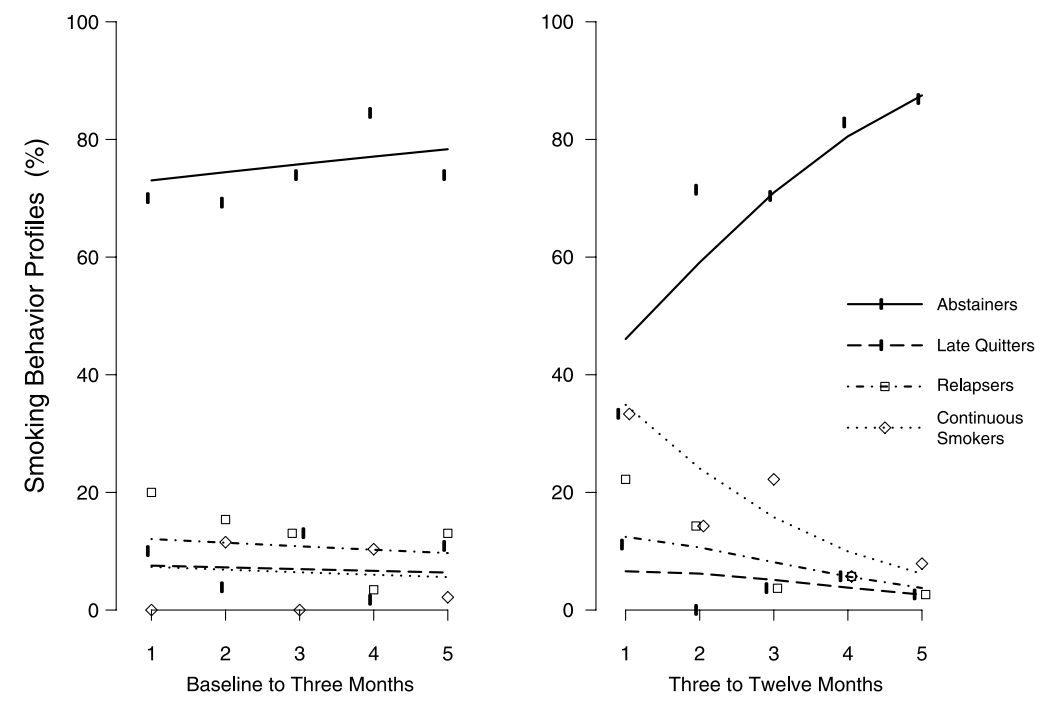

Cancer-Related Risk Perceptions IF CONTINUE SMOKING

Fig. 1. Behavioral motivation hypothesis. Results from the multinomial regression models are plotted as lines, along with the observed proportions plotted as points. On the left panel, the multinomial model estimates the probability of smoking behavior profiles between baseline and three months as a function of the respondents' baseline risk perception. At this time period, there is no association between risk perception and smoking behavior. On the right panel, the model estimates the probability of smoking behavior profiles between three and 12 months as a function of the respondents' 3 month risk perception. Here, there is a strong association between risk perception and smoking behavior. 


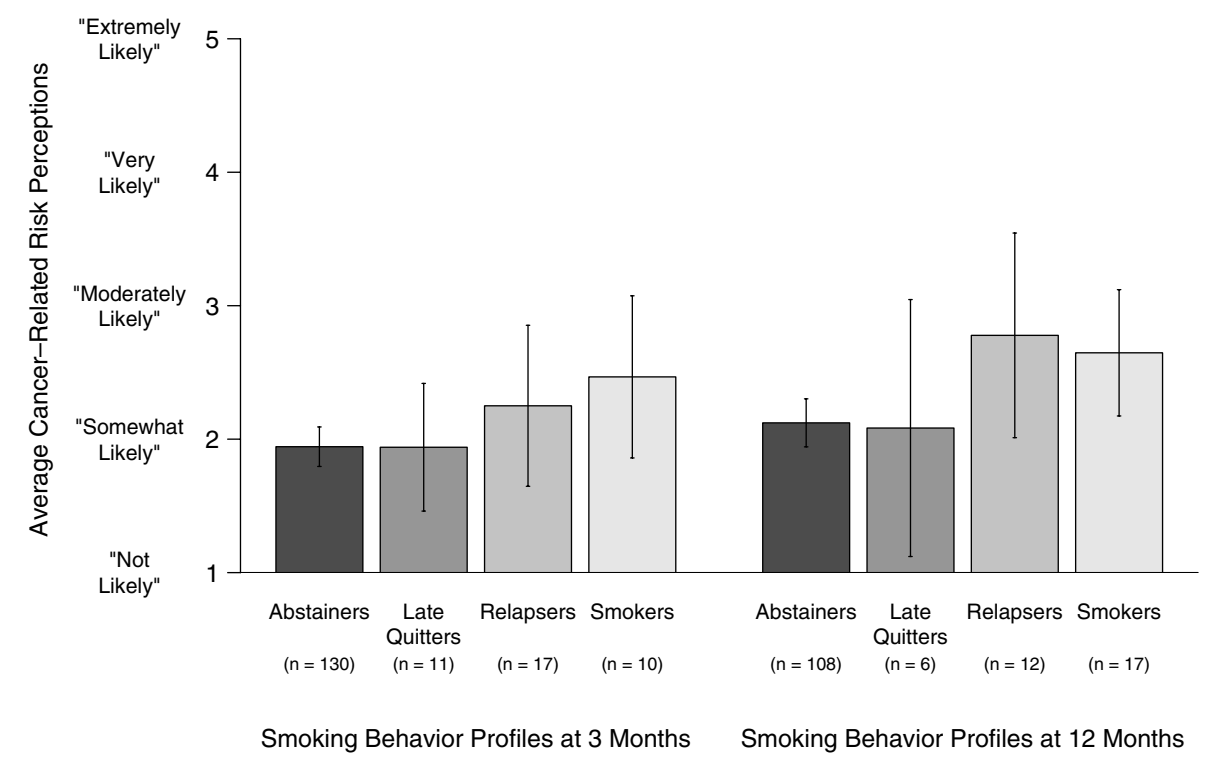

Fig. 2. Accuracy hypothesis. Cancer-related risk perception scores are plotted across smoking behavior profiles at different time periods. The error bars are the $95 \%$ confidence intervals of the mean scores. At 3 months follow-up (left panel), the late quitters were similar to abstainers in their self-appraisal of cancer risks, while relapsers and continued smokers gave slightly higher estimates (not statistically significant by repeated measures ANOVA). At 12 months follow-up (right panel), the trend became statistically significant.

3 months follow-up reliably predicted smoking behavior at 12 months follow-up $\left(\chi^{2}[3, N=125]=\right.$ $10.55, p=0.01)$. Individuals who reported higher perceived risk were significantly more likely to be abstainers than to be continuous smokers or relapsers (abstainers vs. continuous smokers: $\mathrm{OR}=2.08$ with $\mathrm{CI}=[1.22,3.57]$; abstainers vs. relapsers: $\mathrm{OR}=3.03$ with $\mathrm{CI}=[1.42,6.77]$; abstainers vs. late quitters: $\mathrm{OR}=2.22$ with $\mathrm{CI}=[0.70,6.77])$.

We next examined whether baseline stage of disease, baseline Fagerstrom score, baseline cigarettes per day, or other physical status variables (physical symptoms, presence of cancer diagnoses or other medical events at 12 months) affected the significant relationship between cancer-related risk perception at three months and subsequent smoking profile. To do this, we added them as additional simultaneous predictors in the multinomial logistic regression. None of these covariates contributed significantly to 12 month smoking profile (all $p$ s $>.05$ ).

\section{Accuracy Hypothesis}

We next examined whether risk perceptions accurately reflect risk behavior. Figure 2 plots the re- spondents' risk perceptions across smoking profiles at different time periods. The bars on the left are the risk perceptions and smoking behavior profiles at 3 month follow-up, and the bars on the right are the risk perceptions and smoking behavior profiles at 12 month follow-up. The error bars are 95\% confidence intervals of the mean risk perceptions scores. Figure 2 shows that, at 3 months, abstainers reported an average perceived risk of 1.9 (almost a 2.0, a "Somewhat Likely" response). Relapsers and smokers reported slightly higher perceived risk, at 2.3 and 2.5 , respectively. However, as can be gauged by the overlapping confidence intervals, these differences were not statistically significantly different by a univariate analyses of variance (ANOVA; $F[3,157]=$ $1.06, p=0.37)^{5}$

The right panel of Fig. 2 shows that, at 12 months, abstainers reported an average perceived risk of 2.1 (slightly above "Somewhat Likely"), while relapsers and smokers reported higher perceived risk at 2.8 and 2.6, respectively (3.0 represents a "Moderately Likely" response). A second

\footnotetext{
${ }^{5}$ We did not test the accuracy hypothesis at baseline because we did not have available data to calculate smoking behavior profiles at that time period.
} 
ANOVA showed that these averages were statistically different and thus supported the accuracy hypothesis $(F[3,138]=3.2, p=0.03)$. Fisher's Least Significant Difference pairwise tests indicated that abstainers reported significantly lower cancer-related risk perceptions at 12 months than either continuous smokers or relapsers $(p<0.05)$. We next examined whether baseline stage of disease, baseline Fagerstrom score, baseline cigarettes per day, physical symptoms, cancer diagnoses, or other medical events at 12 months influenced 12-month cancerrelated risk perception over and above smoking behavior profile. The accuracy hypothesis remained significant above and beyond the impact of the entered covariates $(F[3,107]=3.07, p=0.03)$. Those who had a new cancer diagnosis between three and 12 months had higher risk perceptions than those who did $\operatorname{not}(F=[1,107]=12.3, p=0.001)$. Baseline stage of disease, physical symptoms, Fagerstrom scores, cigarettes per day, and other medical events were not significantly related to cancer-related risk perceptions at 12 months $(p s>0.05)$.
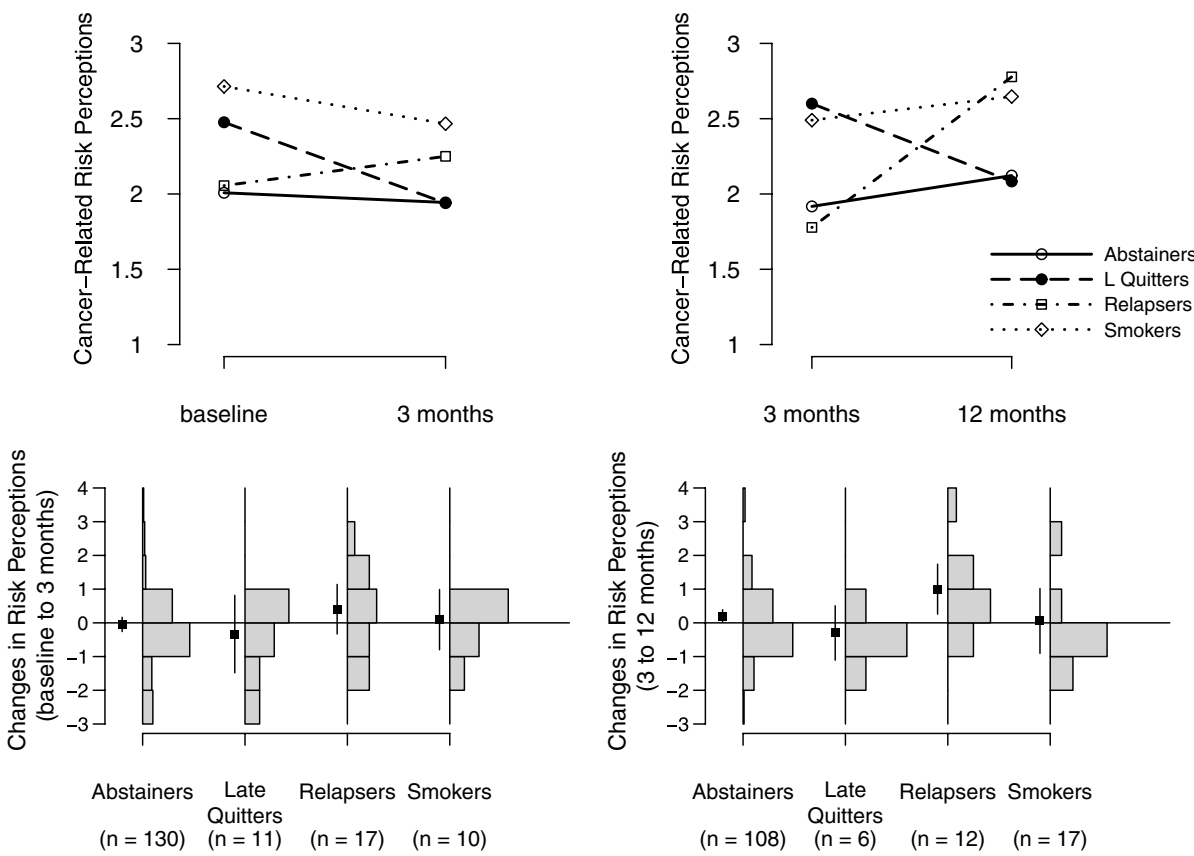

Fig. 3. Risk reappraisal hypothesis. Average risk perceptions scores are plotted over time, separated by smoking behavior profiles. To better understand the individual differences in risk reappraisal over time, the change scores are plotted sideways in the histograms in the bottom panels. To the left of each histogram, the average change scores are plotted as filled squares with the accompanying $95 \%$ confidence intervals as error bars. Note that at 12 months, relapsers significantly increased their reappraisals of cancer recurrence risks. This can be seen in the $95 \%$ confidence interval above the null reduction line, and is supported by repeated-measures ANOVA. 
histograms at the lower right panel show that at 12 months, relapsers tended to shift their risk perceptions up, while those in other smoking behavior profiles did not.

Finally, we examined whether baseline stage of disease, baseline Fagerstrom score, baseline cigarettes per day, physical symptoms, cancer diagnoses, or medical events influenced risk reappraisal at 12 months by adding these variables as simultaneous covariates in the repeated measures ANOVA. The interaction term (risk perceptions across time by smoking behavior profile at 12 months) remained significant $(F[3,102]=4.82, p=0.004)$. Additionally, there was a significant interaction term for risk perceptions across time by cancer events $(F[1,102]=$ $6.9, p=0.01)$ and for risk perceptions across time by cigarettes per day $(F[1,102]=6.3, p=0.01)$, such that people who reported a cancer event and greater baseline numbers of cigarettes per day also reported an increase in cancer-related risk perceptions from three to 12 month assessment.

\section{DISCUSSION}

This study evaluates three discrete hypothesized longitudinal relationships concerning cancer risk perceptions and biochemically-confirmed smoking patterns in a sample of patients newly diagnosed with head, neck, and lung cancers over 12 months. The findings confirm the importance of testing these hypotheses through separate analyses. We found evidence for all three hypothesized relationships (behavior motivation, accuracy, and risk-reappraisal) between cancer-related risk perceptions and postcancer diagnosis smoking patterns between 3 and 12 months. These results were significant despite our modest sample size and $21 \%$ attrition over 12 months. First, cancer-related risk perceptions predicted subsequent quitting behavior (behavior motivation), so that those with higher cancer-related risk perceptions at 3 months were most likely to subsequently abstain from smoking, rather than to smoke continuously. Second, those with different post-diagnosis smoking profiles reported different levels of risk perceptions (accuracy). Relapsers appeared to be particularly sensitive to their risks, as those who relapsed to smoking by 12 months reported the highest cancer-related risk perceptions. Finally, changes in risk perceptions were dictated by smoking behavior profiles (risk reappraisal), as patients who relapsed by 12 months showed the greatest increase in their risk perceptions at 12 months.
These findings are consistent with extensive prior work establishing that heightened illness risk perceptions are a motivator of subsequent behavior change in diverse contexts (Gerrard et al., 1996a, 1996b; Vernon, 1999), including smoking cessation with asymptomatic individuals (Norman et al., 1999). Our current findings are also consistent with work establishing that smokers are relatively accurate in their risk perceptions for developing tobacco-related cancer, rating themselves at higher risk for illness than do nonsmokers (Weinstein, 1999). In contrast, however, Schnoll and colleagues (2002) found that head and neck and lung cancer patients who had quit smoking had higher health risk perceptions than those who continued to smoke. Finally, our findings are consistent with prior literature confirming that changes in risk behaviors lead to subsequent changes in perceptions of risk. For example, Gibbons and colleagues (1991) found that smoking cessation clinic patients who successfully quit after a group intervention subsequently reduced their perceptions of risk for developing smoking-related illnesses (heart disease, emphysema, cancer); relapsers reduced their perceptions of risk to an even greater extent, indicating some defensive denial.

However, the relationships described above were not evident for the earlier time period, which included the period immediately post-surgery through 3 months, indicating the value of assessing these patients over a longer time period. This is not consistent with recent work (Schnoll et al., 2003) which showed that cancer patients' cancer-related risk perceptions were related to smoking cessation at 3 months. This may be due to the fact that Schnoll and colleagues assessed perceptions of a broad range of non-cancer related health risks of continued smoking, as well as perceived risk of cancer recurrence, in their six-item measure of risk perceptions. Indeed, more acute smoking-related risks for surgical complications, treatment side effects, and slowed rate of recovery may be more important motivators of cessation during cancer treatment, with risk of recurrence emerging only later as a predictor of maintenance of tobacco cessation. The potential predictive value of other perceived risks of continued smoking warrant examination in future studies. Conceptually, these findings reinforce the importance of cancer diagnosis as a "teachable moment" for smoking cessation, but caution that cancer risk perceptions may be a more important mechanism for smoking cessation maintenance (e.g., McBride et al., 2003). Additionally, physician advice, social support for quitting 
or psychological distress may be more important predictors of cessation during acute cancer treatment.

Another important study finding involves the fact that levels of nicotine dependence and physical status variables (disease stage, physical symptoms, and medical events) did not change the hypothesized relationships between cancer-related risk perceptions and post-diagnosis smoking pattern. Those who experienced a new cancer event felt at higher risk than those who did not; even so, smoking profile remained an important predictor of cancer-related risk perceptions. Similarly, those who experienced a new cancer event and those who smoked a greater number of cigarettes per day at baseline increased their risk perceptions over time, yet smoking profile continued to significantly predict changes in risk perceptions over time. In contrast with prior literature (Gibbons et al., 1991), our findings do not point to particular defensive denial or cognitive dissonance on the part of those patients who continue to smoke. Relapsers and continuous smokers both assessed higher 12 month cancer risk than those who had quit, and relapsers showed the greatest increase in risk perceptions at 12 months. Perhaps defensive denial is a more plausible response to smoking relapse in smokers not affected by cancer, such as those assessed by Gibbons and colleagues (1991). Relapsers in the current study had already experienced a smoking-related health event that could limit defensive denial of future risk. Despite the fact that those who continued to smoke were relatively accurate concerning their cancer risk, it is certainly possible that there were ways that they under-appreciated the risks that went unmeasured in the current study. These findings point to the importance of continued research addressing health cognitions in smoking cessation and the determinants of long term maintenance of cessation after important health events.

The study findings also provide important justification for continuing attention to the multiple important relationships between risk perceptions and smoking cessation in other groups of smokers, including asymptomatic smokers. Here, the literature is mixed, with some studies indicating a positive relationship between risk perceptions and quitting intentions (Curry et al., 1997; Norman et al., 1999; Strecher et al., 1985), and some studies failing to find the expected positive relationship (e.g., Kirscht et al., 1989; Milam et al., 2000). The use of cross-sectional designs, and a failure to examine the influence of smoking cessation on subsequent risk perceptions, could potentially account for discrepant findings.
We note limitations of the current study. First, we utilized a predominantly Caucasian, highly educated sample of cancer patients; therefore, findings may not generalize to the total population of cancer patients in the United States. In some cases where the cell sizes may drop to below 20, our findings are relatively unstable and may depart from the best estimates we report. In such cases confidence intervals are plotted to indicate the wide margin of errors. The examination of risk perceptions and smoking cessation in racially and ethnically diverse cancer patients is an important next step in this research, especially because diverse cultural and ethnic approaches to risk perceptions are understudied (e.g., Weber and Hsee, 2000). Second, measurement strategies for assessing risk perceptions have been widely criticized (Stefanek et al., 2004), and thus the questions utilized here may not fully capture participants' perceptions of their risk of cancer recurrence. Our dependent variable (post-diagnosis smoking patterns) was calculated based on repeated 7-day point abstinence, which could have resulted in us designating as "abstinent" those individuals who, while having biochemically-confirmed abstinence at 2 time points, could have had a slip to smoking within the designated time period. However, the smoking behavior profiles used in the present study were modeled on previous published work (Ostroff et al., 2000; Schnoll et al., 2003), are amenable to biochemical verification, and, consistent with current clinical treatment practice, provide some leeway for "slips" between assessments that are not considered to be full-blown relapses. Finally, we did not pilot test our cancer risk perception measures with cancer patients, in particular.

Despite these limitations, this study adds to a small literature prospectively examining the relationship of health cognitions and smoking cessation over time in the cancer context. Clinically, the study justifies characterization of smoking cessation after cancer diagnosis as a dynamic, interactive process where, after the most immediate diagnosis and treatment period, cancer-related risk perceptions motivate abstention from smoking, and abstention from smoking reduces risk perceptions over time. These reductions in risk perceptions among quitters may be useful in reducing worries regarding possible disease recurrence. Relapsers stand out as being particularly sensitive to their risks of recurrence, reporting the highest cancer related risk perceptions at 12 months, and the most increase in risk perceptions from 3 to 12 months. This may be because relapsers' 
behavior patterns were counter to their desires and intentions to avoid smoking, and thus they could perhaps be encouraged to make a subsequent quit attempt. As well, this study confirms the theoretical importance of risk perceptions as an important area of investigation in cancer prevention and control. Future prospective assessments of risk perceptions and cancer risk behavior change could help us understand negative findings between risk perceptions and behavior change, expand existing health behavior theories, and develop more nuanced health behavior change interventions.

\section{ACKNOWLEDGMENTS}

This work was supported by NIH grants R29 CA70830, T32 CA0009461, and K07 CA098106. We thank Jane Gooen-Piels, Ph.D., Peggy Maher, Ph.D., Sunita Mohabir, M.A., Valerie Rusch, M.D., and Ashok Shaha M.D. for their support in conducting the study, as well as Jennifer Ford, Ph.D. and Kevin McCaul, Ph.D., for their helpful comments on the manuscript.

\section{REFERENCES}

Agresti, A. (2002). Categorical data analysis, 2nd ed. Wiley, New York.

Aiken, L. S., Fenaughty, A. M., West, S. G., Johnson, J. J., and Luckett, T. L. (1995). Perceived determinants of risk for breast cancer and the relations among objective risk, perceived risk, and screening behavior over time. Women's Health 1: 27-50.

Ajzen, I., and Fishbein, M. (1980). Understanding attitudes and predicting social behavior. Prentice Hall, New Jersey.

Bandura, A. (1986). Social foundations of thought and action: A Social Cognitive Theory. Prentice Hall, New Jersey.

Brewer, N. T., Weinstein, N. D., Cuite, C. L., and Herrington, J. (2004). Risk perceptions and their relation to risk behavior. Ann. Behav. Med. 27: 125-130.

Curry, S. J., Grothaus, L., and McBride, C. (1997). Reasons for quitting: Intrinsic and extrinsic motivation for smoking cessation in a population-based sample of smokers. Addict. Behav. 22: 727-739.

Fagerstrom, K. O., Heatherton, T. F., and Kozlowski, L. T. (1990). Nicotine addiction and its assessment. Ear, Nose, Throat J. 69: 763-765.

Gerrard, M., Gibbons, F. X., Benthin A., and Hessling, R. M. (1996a). A longitudinal study of the reciprocal nature of risk behaviors and cognitions in adolescents: What you do shapes what you think and vice versa. Health Psychol. 15: 344354.

Gerrard, M., Gibbons, F. X., and Bushman, B. J. (1996b). Does perceived vulnerability to HIV motivate precautionary sexual behavior? A critical review of the literature. Psychol. Bull. 119: 390-409.
Gibbons, F. X., McGovern, P. G., and Lando, H. A. (1991). Relapse and risk perception among members of a smoking cessation clinic. Health Psychol. 10: 42-45.

Heatherton, T. F., Kozlowski, L. T., Frecker, R. C., and Fagerstrom, K. O. (1991). The fagerstrom test for nicotine dependence: A revision of the fagerstrom tolerance questionnaire. Br. J. Addict. 86: 1119-27.

Horowitz, A. M., Nourjah, P., and Gift, H. C. (1995). U.S. adult knowledge of risk factors and signs of oral cancers: 1990. $J$. Am. Dental Assoc. 126: 39-45.

Hughes, J. R., Keely, J. P., Niaura, R. S., Ossip-Klein, D. J., Richmond, R. L., and Swan, G. E. (2003). Measures of abstinence in clinical trials: Issues and recommendations. Nicotine Tobacco Res. 5: 13-25.

Hsu, J. C. (1996). Multiple comparisons: Theory and methods. Chapman \& Hall, London.

Janz, N. K., and Becker, M. H. (1984). The health belief model: A decade later. Health Educ. Q. 11: 1-47.

Kirscht, J. P., Janz, N. K., and Becker, M. H. (1989). Psychosocial predictors of change in cigarette smoking. J. Appl. Soc. Psychol. 19: 298-308.

Lichtenstein, E., and Mermelstein, R. J. (1986). Some methodological cautions in the use of the tolerance questionnaire. Addict. Behav. 11: 439-442.

Leventhal, H., and Cameron, L. (1987). Behavioral theories and the problem of compliance. Patient Educ. Couns. 10: 117138.

McCullagh, P., and Nelder, J. A. (1999). Generalized linear models, 2nd ed. Chapman \& Hall, New York.

McBride, C. M., Emmons, K. M., and Lipkus, I. M. (2003). Understanding the potential of teachable moments: The case of smoking cessation. Health Educ. Res. 18: 156-170.

McBride, C. M., and Ostroff, J. S. (2003). Teachable moments for promoting smoking cessation: The context of cancer care and survivorship. Cancer Control 10: 325-333.

Milam, J. E., Sussman, S., Ritt-Olson, A., and Dent, C. W. (2000). Perceived invulnerability and cigarette smoking among adolescents. Addict. Behav. 25: 71-80.

Norman, P., Conner, M., and Bell, R. (1999). The theory of planned behaviour and smoking cessation. Health Psychol. 18: 89-94.

Ostroff, J., Garland, J., Moadel, A., Fleshner, N., Hay, J., Cramer, L., et al. (2000). Cigarette smoking patterns in patients after treatment of bladder cancer. J. Cancer Educ. 15: 86-90.

Portenoy, R. K., Thaler, H. T., Kornblith, A. B., Lepore, J. M., Friedlander-Klar, H., Coyle, N., et al. (1994). Symptom prevalence, characteristics and distress in a cancer population. $Q$. Life Res. 3: 183-189.

Rogers, R. W. (1983). Cognitive and physiological processes in attitude change 1983. A revised theory of protection motivation. In Cacioppo, J., and Petty, R. (Eds.), Social Psychophysiology. Guilford Press, New York, pp. 153-176.

Ronis, D. L. (1992). Conditional health threats: Health beliefs, decisions, and behaviors among adults. Health Psychol. 11: 127134.

Schnoll, R. A., James, C., Malstrom, M., Rothman, R. L., Wang, H., Babb, J., et al. (2003). Longitudinal predictors of continued tobacco use among patients diagnosed with cancer. Ann. Behav. Med. 25: 214-221.

Schnoll, R. A., Malstrom, M., James, C., Rothman, R. L., Miller, S. M., Ridge, J. A., et al. (2002). Correlates of tobacco use among smokers and recent quitters diagnosed with cancer. Patient Educ. Couns. 46: 137-145.

Sidak, Z. (1967). Rectangular confidence regions for the means of multivariate normal distributions. J. Am. Statist. Assoc. 62: 626-633.

Stefanek, M., Weinstein, N., Cameron, L., McCaul, K., Rayna, V. F., Croyle, R., et al. (2004). State of the art in risk perception research. Ann. Behav. Med. 27: S001. 
Strecher, V. J., Becker, M. H., Kirscht, J. P., Eraker, S. A., and Graham-Tomasi, R. P. (1985). Psychosocial aspects of changes in cigarette-smoking behavior. Patient Educ. Couns. 7: 249-62.

Sutton, S. R. (1982). Fear-arousing communications: A critical examination of theory and research. In Eiser, J. R. (Ed.), Social Psychology and Behavioral Medicine. Wiley, Chichester, pp. 303-338.

Vernon, S. (1999). Risk perception and risk communication for cancer screening behaviors: A review. J. Natl. Cancer Inst. 25: 101-118.

Warnakulasuriya, K. A. A., Harris, C. K., Scarrott, D. M., Watt, R., Gelbier, S., Peters, T. J., et al. (1999). Oral Cancer-a survey of the general public. Br. Dental J. 187: 319-322.
Weber, E. U., and Hsee, C. K. (2000). Culture and individual judgment and decision-making. J. Appl. Psychol. 49: 32-61.

Weinstein, N. D. (1988). The precaution adoption process. Health Psychol. 7: 355-386.

Weinstein, N. D. (1999). Accuracy of smokers' risk perceptions. Nicotine Tobacco Res. 1(Suppl. 1): 123-130.

Weinstein, N. D., and Nicolich, M. (1993). Correct and incorrect interpretations of correlations between risk perceptions and risk behaviors. Health Psychol. 12: 235-245.

Weinstein, N. D., Rothman A. J., and Nicolich, M. (1998). Use of correlational data to examine the effects of risk perception on precautionary behaviors. Psychol. Health 13: 479501. 